\title{
Vertical datum unification for the International Height Reference System (IHRS)
}

Laura Sánchez, Deutsches Geodätisches Forschungsinstitut, Technische Universität München, Arcisstr. 21, 80333 München, Germany, Im.sanchez@tum.de Michael G. Sideris, Department of Geomatics Engineering, University of Calgary, 2500 University Drive NW, Calgary, Alberta, T2N 1N4, Canada, sideris@ucalgary.ca

Vertical datum parameters

The International Association of Geodesy (IAG) released in July 2015 a resolution for the definition and realisation of an Internationa Height Reference System (IRRS). According to this resolution, the HRS coordinates are potential differences referring to the level urface of the Earth's gravity field realised by the conventional value $W_{0}=62636853.4 \mathrm{~m}^{2} \mathrm{~s}^{-2}$. A main component of the IHRS is the integration of the existing height systems into the global one; i.e., reference known as vertical datum unification and its main result are the vertical datum parameters ie, the potential differences betwe the local and the global reference levels (Fig. 1):

$\delta W_{0 i}=W_{0}-W_{0 i}$

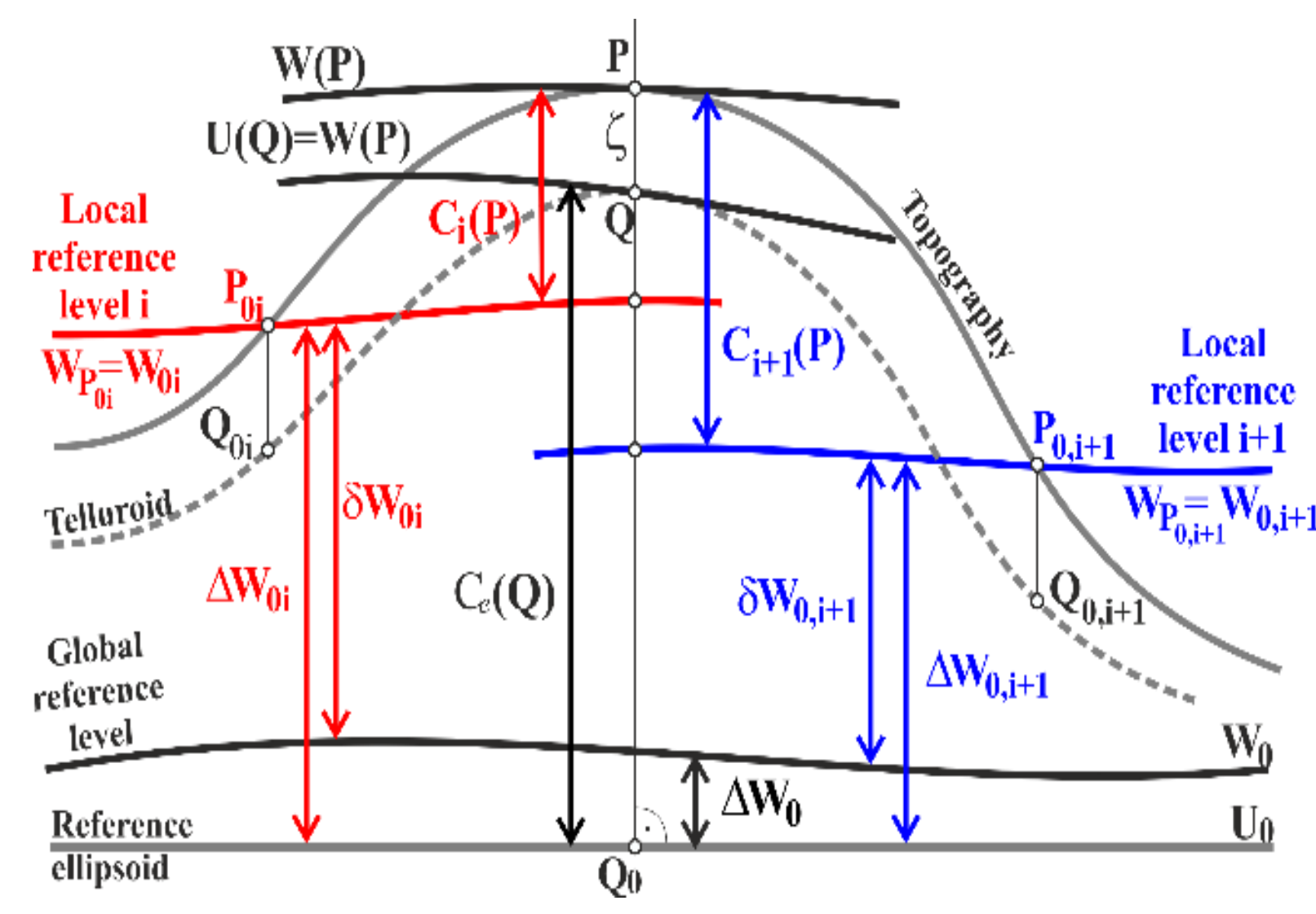

Fig. 1: Vertical datum parameters for the local height systems $i$ and it $l$.

Observation equations for the vertical datum unification The estimation of the vertical datum parameters is based on the comparison of the height anomaly $\zeta$ (or geoid undulation $N$ ) obtained from the solution of the geodetic boundary value problem (GBVP)
with the height anomaly $\zeta$ (or geoid undulation $N$ ) derived from combining satellite-based ellipsoidal heights $(h)$ with levelling-based geopotential numbers $(C)$ or physical heights $\left(H^{*}\right.$ or $\left.H\right)$. For a general formulation, independent of normal or orthometric heights, the observation equations are given here in terms of potential

$l_{P}=h(P) \gamma-C_{i}(P)-T_{i}(P)+\Delta W_{0}=$

$\left(1+f_{0 i}(P)\right) \delta W_{0 i}(P)+\sum_{j=0 j}^{J} f_{0 j}(P) \delta W_{0 j}(P)+v_{P}$

At each point $P$ referring to the vertical datum $i$, an observation equalion like Eq. [2] can be formulated. Al those poins referring to

$$
l_{P}=C_{i+1}(P)-C_{i}(P)=\delta W_{0 i}-\delta W_{0, i+1}+v_{P}
$$

Least-squares estimation of the vertical datum parameters

Equations [2] and [4] can be solved by a least-squares adjustment. The functional and stochastic models are given by:

$$
\mathbf{A} \mathbf{x}-\mathbf{v}=\mathbf{l}
$$

$E\{\mathbf{v}\}=0 ; \quad E\left\{\mathbf{v v}^{T}\right\}=\mathbf{C}_{l}=\mathbf{C}_{h(P)}+\mathbf{C}_{C_{i}(P)}+\mathbf{C}_{T_{i}(P)}$

$A$ is the design matrix containing the coefficients of the unknowns in the observation equations; $\mathbf{x}$ is the vector of the unknowns; $\mathbf{v}$. Eqs. [2] and [4]; $E\{\boldsymbol{\varphi}\}$ is the expectation operator; $\mathbf{C}$ represents the

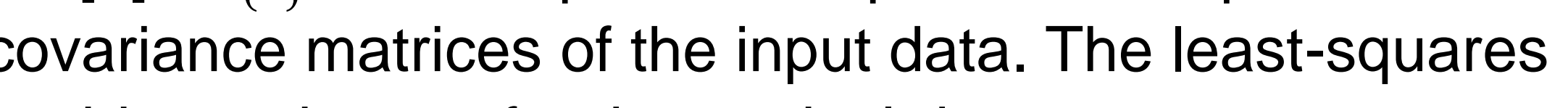
solution provides estimates for the vertical datum parameters $\delta W_{0 i}$ and the corresponding variance-covariance matrix:

$\mathbf{x}=\left(\mathbf{A}^{T} \mathbf{C}_{l}^{-1} \mathbf{A}\right)^{-1}\left(\mathbf{A}^{T} \mathbf{C}_{l}^{-1} \mathbf{l}\right) ; \quad \mathbf{C}_{\mathbf{x}}=\left(\mathbf{A}^{T} \mathbf{C}_{l}^{-1} \mathbf{A}\right)^{-1}$

Effect of omission error and indirect bias term on datum unification in North America

The disturbing potential $T_{i}(P)$ (Eq. [3]) is typically computed by the remove-compute-restore procedure:

$T_{i}(P)=T_{\text {GCiM }}(P)+T_{i, r e s}(P)$

Eq. [8]

$T_{G G M}(P)$ is inferred form a global gravity model (GGM) of maximum degree and order $N_{\max } \cdot M_{i, r e s}(P)$ is estimated by evaluating Eq. [3] using residual gravity anomalies $\Delta g_{i, r e s}=\Delta g_{i}-\Delta g_{G G M}$ and the residual kerform acal gravity and topography data, the following quesions a a Q1. will $T_{\text {iress }}(P)$ in Eq. [8], or omission error, be small enough to

Q2. will the last term of the indirect bias in Eq. [2] be small enough to omit?

$\Delta W_{0}=W_{0}-U_{0}$ is the potential difference between the IHRS $W_{0}$ value and the normal potential $U_{0}$ of the GRS80 ellipsoid.

$\delta W_{0 i}$ denotes the vertical datum parameters (cf. Eq. [1]). $T_{i}(P)$ is the usual result obtained by solving the GBVP using (biased) gravity anomalies $\left(\Delta g_{i}\right)$ referring to the vertical datum $T_{i}(P)=\frac{G \delta M}{R}+\sum_{i=1}^{J} \frac{R}{4 \pi} \iint\left(\Delta g_{i}\left(P_{k}\right)+g_{i i}\left(P_{k}\right)\right) S\left(\psi\left(P, P_{k}\right)\right) d \sigma \quad$ Eq. [3]

$\sum^{J} f_{0 j}(P) \delta W_{0 j}(P)+v_{P}$ with $f_{0 i}(P):=\frac{1}{2 \pi j} \iint S\left(\psi_{P, P_{R}}\right) d \sigma$ denotes an indirect bias term caused by the effects of the level differences $\delta W_{0 i}$ on the boundary values (i.e., $\Delta g_{i}$ ).

and $v_{p}$ represent the observables and the stochastic residuals respectively.
Consequently, the $f_{0 i}$ coefficients in Eq. [2] can be set equal to zero, resulting in a much simpler system of observation equatio weighted mean of all individual station $\delta W_{(}(P)$ vals are the weighed mean of all individual station o $w_{0 i}(P)$ values. Fig. 3 to the IHRS $W_{0}$ reference level that were used to obtain the results shown in Table 1 and Fig. 2.

Table 1: Statistics of the DIR5 model omission error computed at tide gauges
using local data. Map shows the geographic location of the tide gauges.
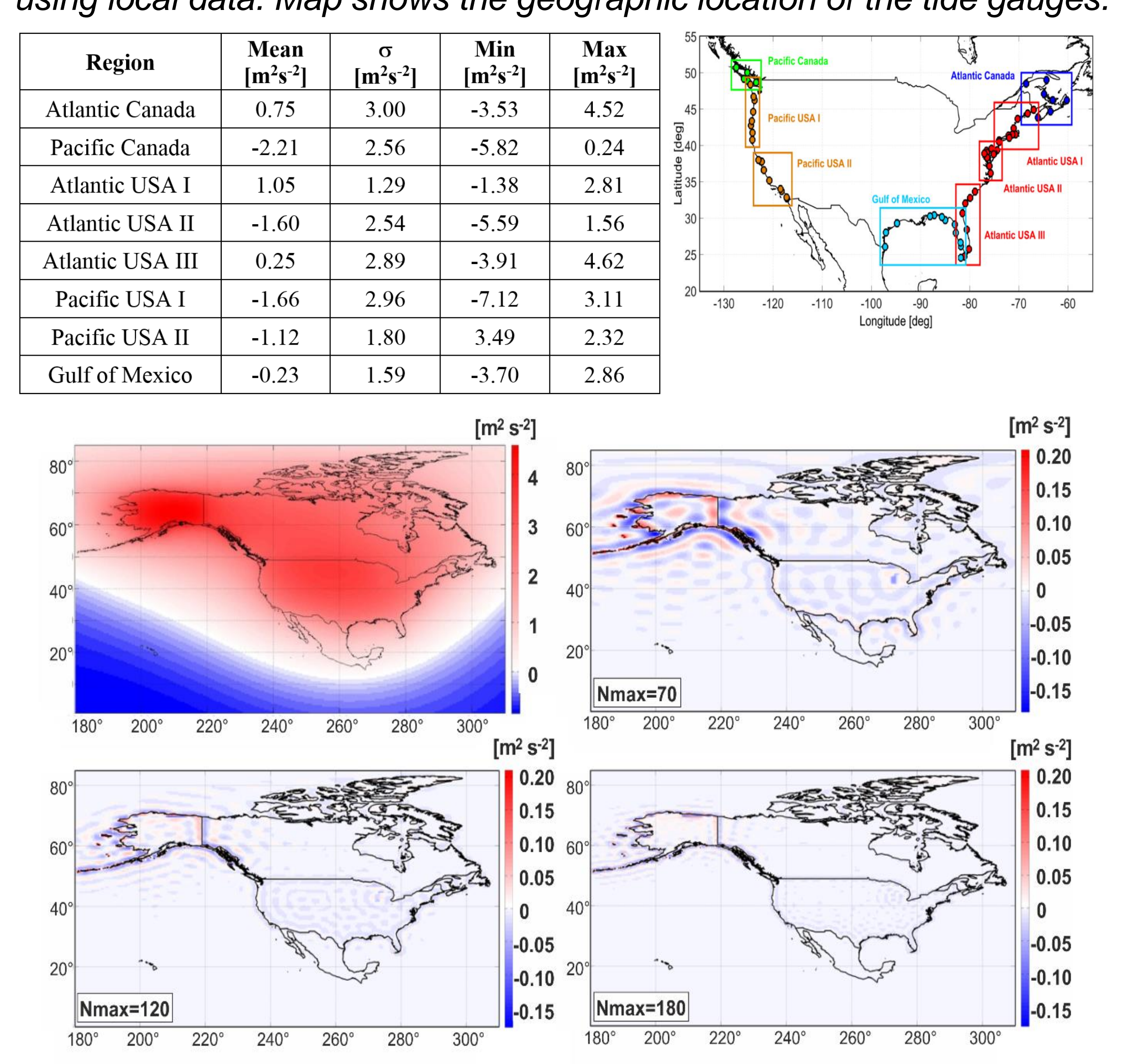

Fig. 2: Indirect bias term computed with the full--unmodified kernel function (leth

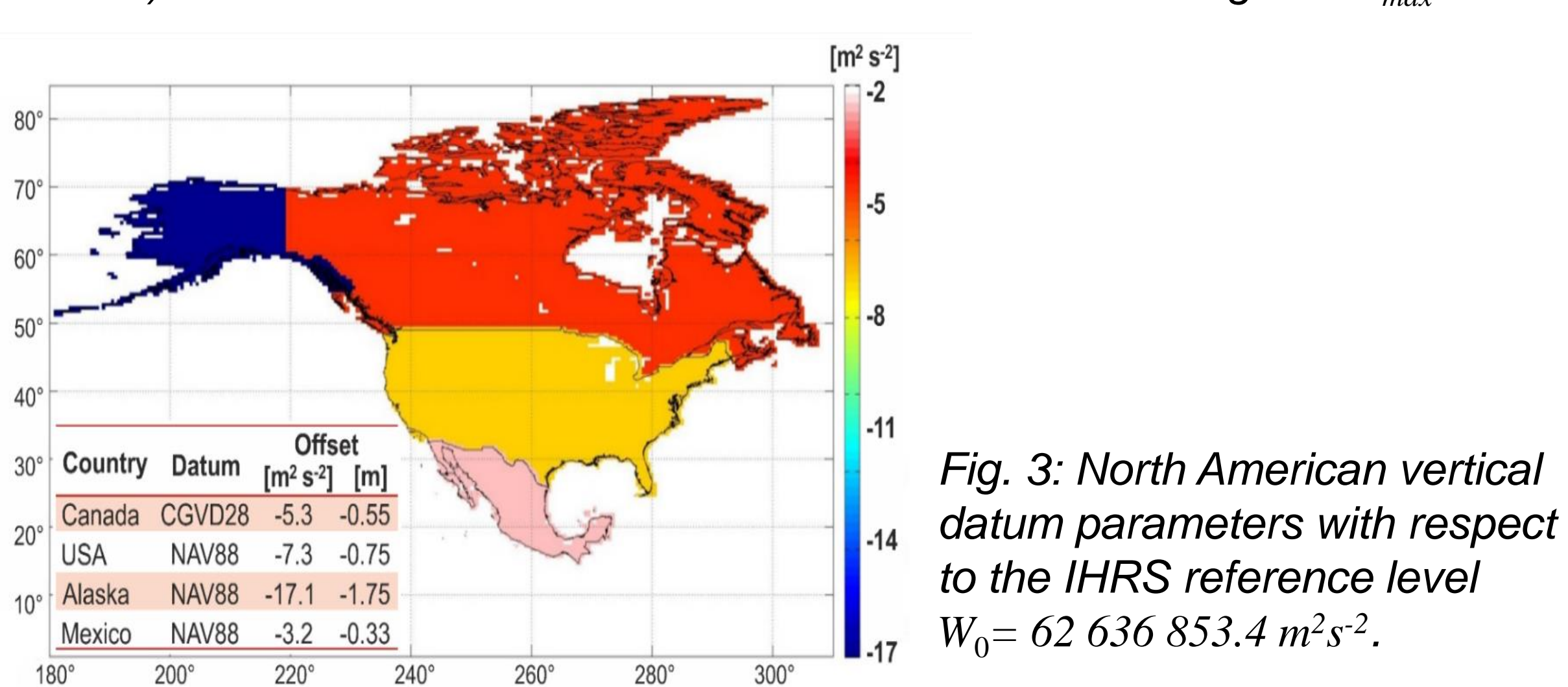

Vertical datum unification in South America

The formulation of the observation equations [2] and [4] implies the avallability of terrestral gravity anomalles, levelling-based geopotentil numbers, ellipsoidal heights from GNSS on land and from satell eallimetry ili oceans, and border levelling points with geopotent to the geodetic data available in South America, this study is based on

14 observation equations of the type Eq. [2] in the marine areas nearby the reference tide gauges (Fig. 4a):

(2) stations of the continental refonge frame SIRGAS (Fig 4b); 7 observation equations of the type Eq [4]: Cong 7 observation equations of the type Eq. [4]. connections Venezuela and Brazil, and Brazil and Argentina (Fig 4C).

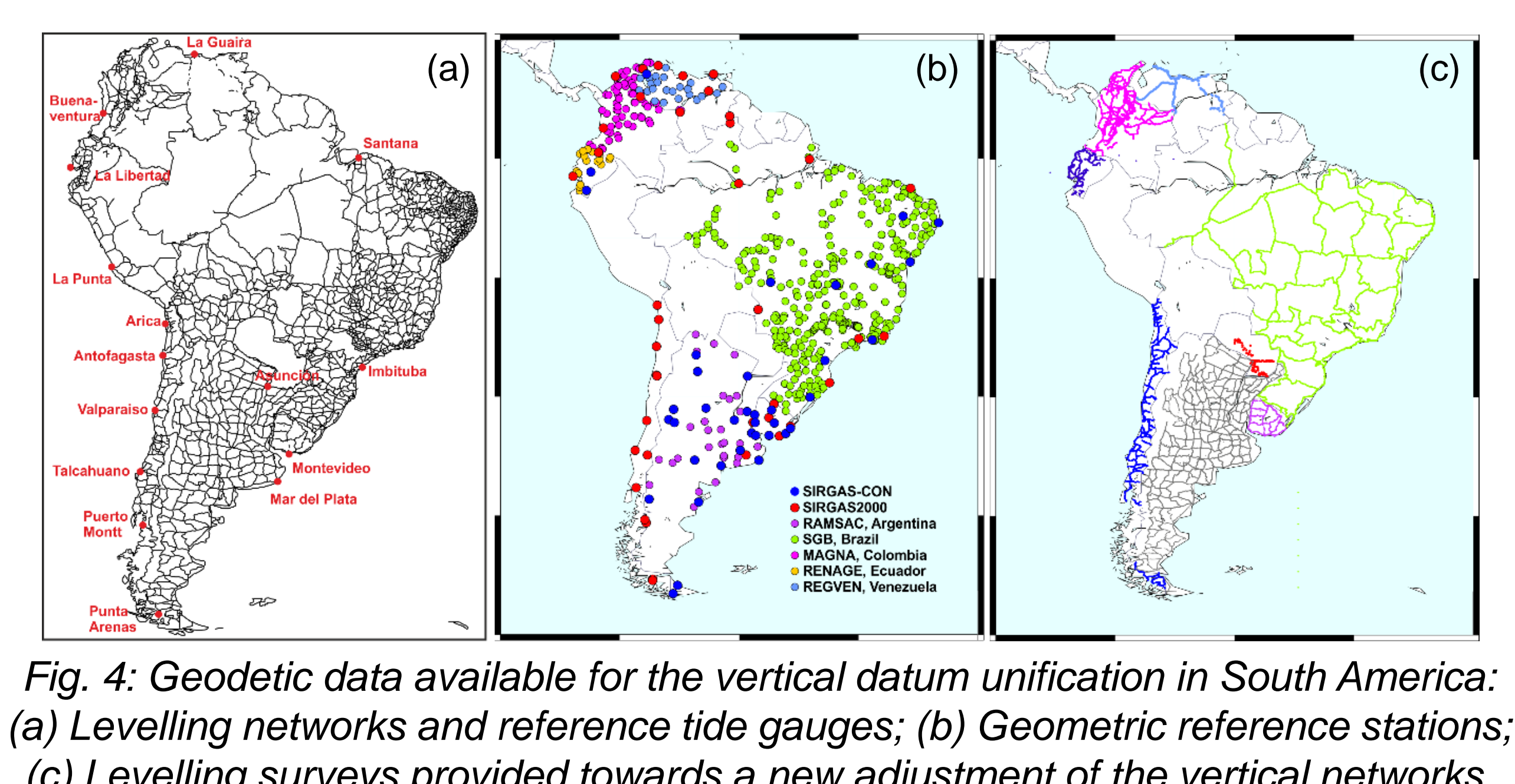

(c) Levelling surveys provided towards a new adjustment of the vertical networs

In a first estimation, Eq. [7] was solved by employing the data ferred to different ITifterent South American countries, i.e., different tide systems, etc. A second estimation was made based on standardised data. The purpose was to compare the vertical datum parameters obtained using the available raw geodetic data (without further processing) and hose parameters obtained with differentsed (or standardised) geodelic data. The accuracy of the coveriabs cosiduals and the estimated vertical datum parameters for the existing South American height systems. The accuracy was assessed to be $+0.5 \mathrm{~m}^{2} \mathrm{~s}^{-2}$ in those regions with a high number of observations; i.e., Argentina, Brazil (Imbituba), Colombia, Ecuador, Uruguay and Venezuela. In regions with a small number of observations, like the northern part of Brazil (Santana), Bolivia, Peru and the southern part of Chile (Punta Arenas), the accuracy was not better than $\pm 2 \mathrm{~m}^{2} \mathrm{~s}^{-2}$ to $\pm 4 \mathrm{~m}^{2} \mathrm{~s}^{-2}$.
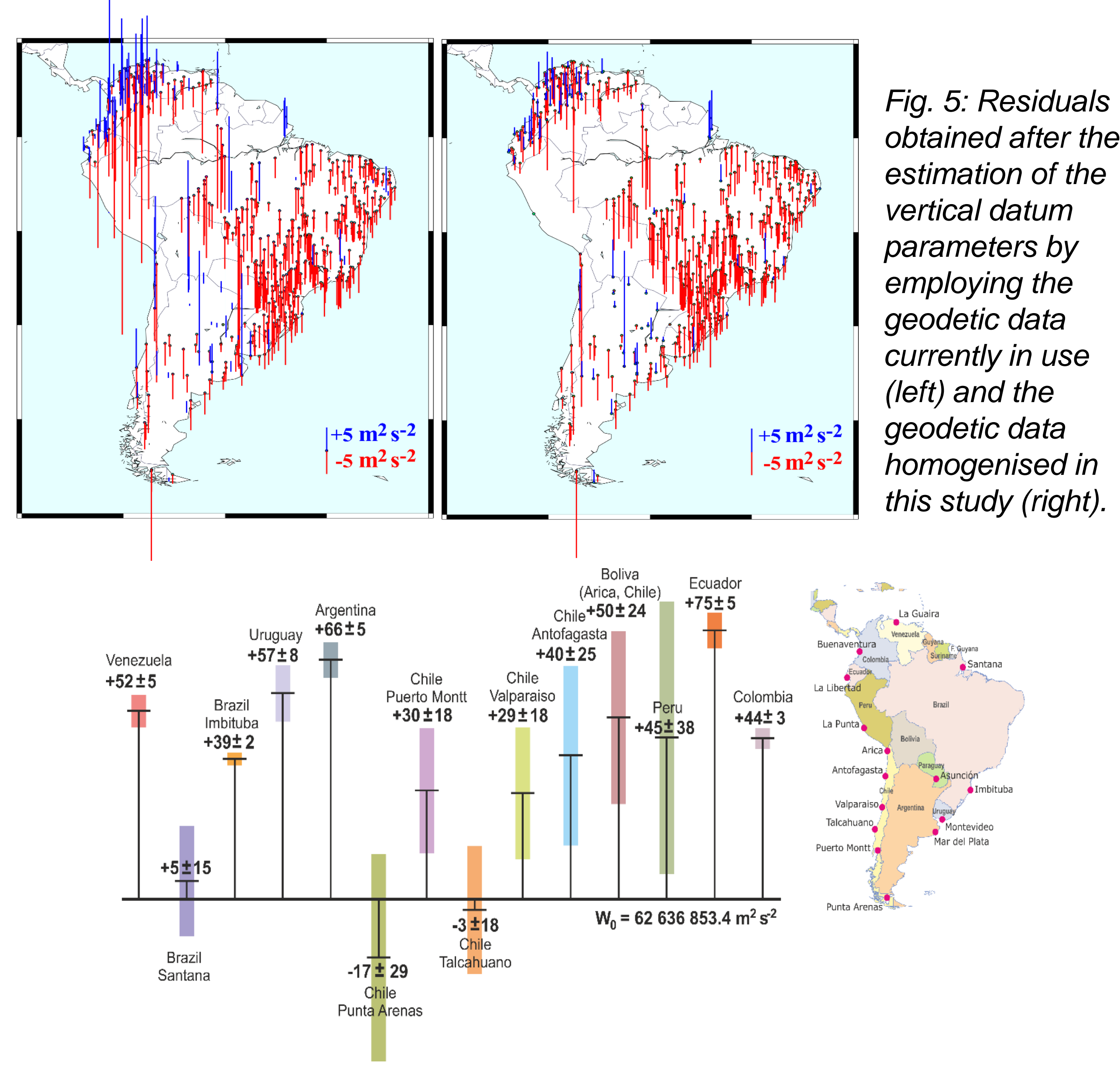

Fig. 6: South American vertical datum parameters [in $\mathrm{m}$ ] with respect to the IHRS
reference level $W_{0}=62 \quad 636853.4 \mathrm{~m}^{2} \mathrm{~s}^{2}$. Coloured bars show the standard deviations Further reading: Sánchez L., Sideris M.G.: Vertical datum unification for the $209(2), 570-586,10.1093 /$ lijilgag 025,2017 